\title{
Development and User Satisfaction of "Plan-It Commander," a Serious Game for Children with ADHD
}

\author{
Kim C.M. Bul, MSc, ${ }^{1,2}$ Ingmar H.A. Franken, $\mathrm{PhD}^{2}$, Saskia Van der Oord, $\mathrm{PhD}^{3-5}$ \\ Pamela M. Kato, PhD, ${ }^{6}$ Marina Danckaerts, $\mathrm{PhD},{ }^{7}$ Leonie J. Vreeke, $\mathrm{PhD}{ }^{1,2}$ \\ Annik Willems, MSc, ${ }^{8}$ Helga J.J. van Oers, $\mathrm{PhD}^{9}{ }^{9}$ Ria van den Heuvel, MSc, ${ }^{10}$ \\ Rens van Slagmaat, B CMD,11 and Athanasios Maras, $\mathrm{MD}^{1}$
}

\begin{abstract}
The need for engaging treatment approaches within mental health care has led to the application of gaming approaches to existing behavioral training programs (i.e., gamification). Because children with attention deficit/ hyperactivity disorder (ADHD) tend to have fewer problems with concentration and engagement when playing digital games, applying game technologies and design approaches to complement treatment may be a useful means to engage this population in their treatment. Unfortunately, gamified training programs currently available for ADHD have been limited in their ability to demonstrate in-game behavior skills that generalize to daily life situations. Therefore, we developed a new serious game (called "Plan-It Commander") that was specifically designed to promote behavioral learning and promotes strategy use in domains of daily life functioning such as time management, planning/organizing, and prosocial skills that are known to be problematic for children with ADHD. An interdisciplinary team contributed to the development of the game. The game's content and approach are based on psychological principles from the Self-Regulation Model, Social Cognitive Theory, and Learning Theory. In this article, game development and the scientific background of the behavioral approach are described, as well as results of a survey $(n=42)$ to gather user feedback on the first prototype of the game. The findings suggest that participants were satisfied with this game and provided the basis for further development and research to the game. Implications for developing serious games and applying user feedback in game development are discussed.
\end{abstract}

\section{Introduction} Support and improve primary care processes in mental health care and are often referred to as e-mental health. ${ }^{1,2}$ Clinicians and educators are interested in applying game technologies and game design approaches (e.g., serious games) because of their potential to increase patient engagement with existing behavioral training programs. ${ }^{3}$ Game elements that increase patient engagement in therapeutic activities have the potential to increase the effectiveness of neurocognitive training and behavioral learning in different domains of functioning for patients being treated in mental health care. ${ }^{4-6}$

Game design and approaches are seen as a natural tool to make existing training and therapeutic programs more appealing to young patients with attention deficit/hyperactivity disorder (ADHD) for several reasons. First, it is well known that children with ADHD experience motivation deficits and

\footnotetext{
${ }^{1}$ Yulius Academy, Yulius Mental Health Care Organization, Barendrecht, The Netherlands.

${ }^{2}$ Department of Clinical Psychology, Erasmus University of Rotterdam, Rotterdam, The Netherlands.

${ }^{3}$ Faculty of Psychology and Educational Sciences, KU Leuven, Leuven, Belgium.

${ }^{4}$ Department of Developmental Psychology, University of Amsterdam, Amsterdam, The Netherlands.

${ }^{5}$ Cognitive Science Centre Amsterdam, University of Amsterdam, Amsterdam, The Netherlands.

${ }^{6}$ Serious Games Institute, Faculty of Engineering and Computing, Coventry University, Coventry, United Kingdom.

${ }^{7}$ Department of Psychiatry, KU Leuven, Leuven, Belgium.

${ }^{8}$ Venture \& Incubation Centre, Janssen Pharmaceuticals, Beerse, Belgium.

${ }^{9}$ Department of Medical Affairs, Janssen Pharmaceuticals, Tilburg, The Netherlands.

${ }^{10}$ Centre ZitStil, Antwerp, Belgium.

${ }^{11}$ RANJ Serious Games, Rotterdam, The Netherlands.
} 
react differently to rewards compared with typically developing children. ${ }^{7,8}$ Because game approaches help to balance motivating and learning elements and to integrate game goals and behavioral/cognitive challenges, they have the potential to keep these children more motivated and positively engaged in therapy processes. ${ }^{9-11}$ Also, despite their poor attention span, distractibility, and difficulty staying on task, children with ADHD often show sustained concentration and engagement when playing digital games. ${ }^{12}$ Therapeutic goals that are pursued in the context of an engaging game environment thus present the opportunity to improve behavioral learning and outcomes in this population.

Large numbers of gamified training programs for ADHD have been designed to improve working memory and executive functioning, thereby addressing specific neurocognitive deficits. ${ }^{13-16}$ Although these programs show some evidence for short-term effects on targeted working memory outcomes, as measured by neurocognitive tests that are similar to the ones presented in the games, they have not shown compelling evidence that these effects generalize beyond neurocognitive outcomes to important domains of functioning in the everyday lives of children with ADHD. ${ }^{13-16}$ The core symptoms of inattention, impulsivity and hyperactivity among children with ADHD are related to their difficulties in executive and social functioning in their daily lives. These problems include difficulties managing time, keeping deadlines, planning/organizing schoolwork, and making friends. ${ }^{17-20}$ These executive functioning and social problems not only affect daily life for the children and their families, but they also predict a poor prognosis of ADHD even into adulthood. ${ }^{20}$ Gamified interventions for children with ADHD that address the current difficulties in daily life functioning thus have the potential to tackle difficulties not only in the short term but in the long term as well. Although the research on gaming approaches to addressing daily life functioning of children is limited, several controlled trials of serious games developed for other patient groups have been shown promise of impacting "real world" behaviors. $^{21}$

In addition to the importance of designing a serious game intervention to impact important outcomes that support their functioning in daily life, the intervention itself needs to be designed to be effective and engaging in order to ultimately have an impact. Previous studies provided evidence that gamified interventions based on theoretical concepts tend to be more effective than those without a theoretical framework. $^{22}$ Integrating appropriate behavioral theories into the design of the game is an ongoing challenge for serious game designers but is a key to its ultimate success. ${ }^{23}$ The focus on integrating behavior change theories and therapeutic content in serious game design needs to be balanced by technology acceptance through the target audience of children with ADHD and their parents who will likely play a key role in accessing, facilitating, and monitoring the use of the serious games technology. A broad body of evidence has shown that the success of information technologies, such as serious games, depends on user beliefs and attitudes about the technology (e.g., "The game performs reliably, and it is easy to interact with this game"), as well as their behavioral beliefs and attitudes about using the system (e.g., "This game helps me understand how I can plan and organize my time"). ${ }^{24}$ Gathering this information is an important part of the development process to provide an intermediate evalu- ation of design decisions and a basis for major or minor design decisions to promote the success of the product. ${ }^{25}$

In this study, we describe the development process of a serious game we developed for children with ADHD that encourages behavioral learning and promotes strategy use in important domains of daily life functioning, namely, time management, planning/organizing, and prosocial skills. We also present results of a user satisfaction survey we conducted on a pilot version of the game.

\section{Theoretical Basis for the Serious Game Intervention}

We developed a serious game called "Plan-It Commander" for a target population of children with ADHD 8-12 years of age. The therapeutic behavioral learning objectives of the serious game were to promote the use of strategies in important domains of daily life functioning, namely, time management, planning/organizing, and prosocial skills. These behavioral objectives were translated into a suitable game based on relevant psychological theories, such as (1) the Self-Regulation Model, ${ }^{26}$ (2) Social Cognitive Theory, ${ }^{27}$ and (3) Learning Theory. ${ }^{12,28}$

The Self-Regulation Model of health and illness behavior focuses on how individuals direct and monitor their activities and emotions in order to attain their goals. ${ }^{12,26}$ Children with ADHD often lack self-regulation, and as a consequence they master skills at a lower level. In addition, they feel incompetent about their performance and think that they cannot cope with situations in which these skills have to be used. The serious game contained components that helped them direct and monitor their activities (e.g., predict how long it would take them to complete a "mission"), regulate their emotions (e.g., slow down to help other characters in the game in order to "win"), and practice as many times as needed in order to reach mastery (e.g., no overt or explicit penalties for "mission failure"). Components such as these were explicitly built into the system to provide a safe environment to practice skills that could be applied in their daily life.

The serious game also included elements from Social Cognitive Theory. ${ }^{27}$ According to this theory, children's learning is influenced by interactions among the environment, personal factors, and behavior. The environment supports mastery of target behaviors by providing models for target behaviors and positive support for behavior change. This theory was translated into the game by offering children with ADHD structured behavioral goals to reach in the game (e.g., collect minerals with the time that you estimate it will take to complete the task). These goals were presented in an environment that included a virtual mentor figure who was a model of positive behavior (e.g., polite in social interactions) and also provided emotional encouragement and positive feedback for success and multiple opportunities to practice behaviors to reach mastery. The game environment also included a social community in which peers (other children with ADHD) could interact with each other. Players could also directly or indirectly benefit from positive reinforcements they observed others received or that they received directly as a result of their own successful efforts to reach goals in the game. The concepts of vicarious learning, emotional support, and provision of mastery experiences, which are key components of behavior change in Social Cognitive Theory, ${ }^{27}$ were implemented in the game design. 
Lastly, principles of Learning Theory were incorporated in the serious game. Learning Theories are based on the general idea that individuals learn behavior through behavioral consequences and positive reinforcement. ${ }^{12,28,29}$ Children with ADHD are less sensitive to negative feedback and learn the most through repetitive positive feedback. In this game we immediately reward positive behavior, based on this principle. As a result, extensive practice of desired behaviors is stimulated.

\section{Collaborative Game Development}

Interdisciplinary collaboration is a key factor in developing a serious (either educative or therapeutic) game, as different expertise from various areas (clinical, research, technical and game design) needs to be integrated. ${ }^{23}$ Therefore, different parties (i.e., sponsor, game development company, healthcare professionals, researchers, and parents and children with ADHD) were involved in the development of the "Plan-It Commander" game. In collaboration with a community board of parents, the learning goals (e.g., time management, planning/organizing, and prosocial skills) were proposed by healthcare professionals based on scientific literature and practical clinical experience. Furthermore, these professionals provided input on the game goals and advised the game designers on how to give feedback to children with ADHD. Frequent interactive sessions among the behavior experts, researchers, and game designers took place to optimize the link between game elements and the principles of behavioral intervention, allowing game designers to gain additional expertise and knowledge to develop an attractive game that "works" for this target population. ${ }^{23}$

Results of important deliverables and milestones were presented to the advisory board, consisting of professionals familiar with the content of gaming, research, and clinical practice. Researchers were involved to design and set up research trajectories to test game usability and effectiveness. After each prototype, usability tests were iteratively performed to examine whether children liked the game, as well as understood how to use it and navigate within the game. These user data were evaluated and incorporated in the design process. Parallel to testing the first prototype in a pilot study, the game was further developed and extended resulting in the final version of the game described in this article. The stages of game development and evaluation are illustrated in Figure 1.

\section{Game Description}

"Plan-It Commander" is an online computer game with a futuristic and adventurous character consisting of two parts:
(1) the mission game (a game environment with missions and three isolated minigames with embedded learning goals) and (2) a closed social community for interaction through predefined messages. Each minigame has levels of increasing complexity and performance challenges. In the game the player is a space captain undertaking missions assigned by his or her mentor who guides the player, gives him or her feedback, and helps wherever he can. The player's goal is to collect and recover rare minerals. Characteristics of "Plan-It Commander" are described in Table 1.

To motivate and engage children throughout the game several special features were designed (Table 2 and Fig. 2).

\section{Missions and side missions}

The game is divided into 10 different missions and several side missions. Missions guide the player's behavior throughout the game as he or she follows the story line and is confronted with assignments requiring specific skills to solve problems. Completing these assignments ensures skills concerning time management, planning/organizing, and prosocial behavior are practiced and trained. Each mission has different tasks, and the player has a mission board to check which missions he or she has completed. Once a mission is completed the next mission becomes available. Side missions are independent missions, separate from the main storyline, and are optional. Several side missions focus on triggering the player's prosocial behavior (e.g., players can ask other players for assistance [e.g., finding special items] and in turn decide whether to provide assistance). In addition, players can make short-term and long-term appointments with other nonplayable characters (e.g., to retrieve items). Further general learning goals throughout the game include listening to the mentor, dealing with frustration, ignoring distraction, learning to concentrate, being attentive, and inhibiting impulses.

\section{Minigames}

A minigame is a small, isolated game within the larger game environment that integrates unique game elements offering tools to improve strategic behavior. Players begin with an explanatory tutorial level and progress through the game by accomplishing levels within the minigames and missions. Three minigames with assignments addressing time management, planning/organizing, and prosocial behavior are embedded in the game (Fig. 3).

Minigame 1: "Labyrinth." Within this minigame the player learns to manage time and to estimate time needed. In

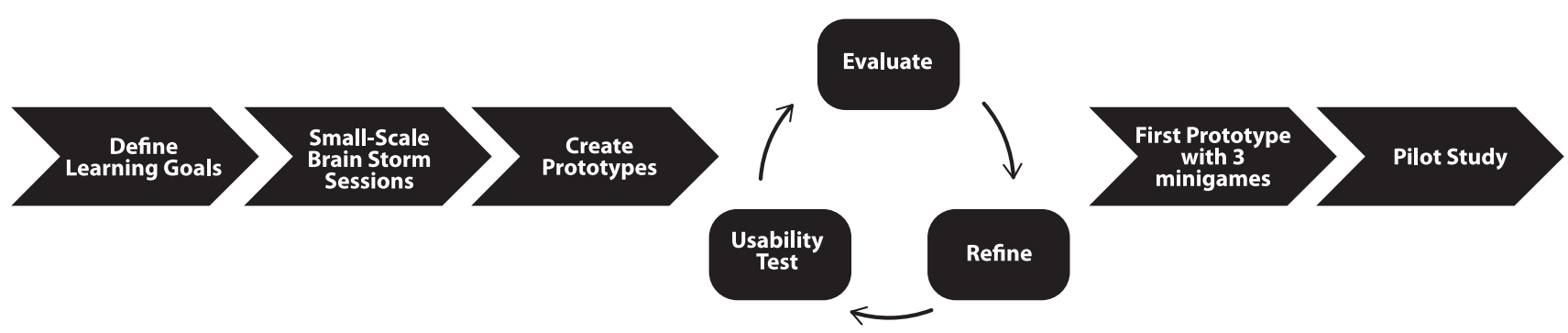

FIG. 1. Stages of game development. 
Table 1. Characteristics of a Videogame for Health: "Plan-It Commander"

Characteristic Focus

Health topic(s)

Targeted age group(s)

Other targeted group characteristics

Short description of game idea

Target player(s)

Guiding knowledge or behavior change theory(ies), models, or conceptual framework(s)

Intended health behavior changes

Knowledge element(s) to be learned

Behavior change procedure(s) (taken from Michie inventory) or therapeutic procedure(s) used

Clinical or parental support needed (please specify)

Data shared with parent of clinician

Type of game

Story

Synopsis (including story arc)

How the story relates to targeted behavior change

Game components

Player's game goal/objective(s)

Rules

Game mechanic(s)

Procedures to generalize or transfer what's learned in the game to outside the game

Virtual environment

Setting (describe)

Avatar

Characteristics

Abilities

Game platform(s) needed to play the game

Sensor used

Estimated play time
Mental health

8-12 years of age

ADHD

A serious game that includes behavioral learning and promotes strategy use in important domains of daily life functioning Individual

Self-Regulation Model, Social Cognitive Theory, and Learning Theory

Time management, planning/organizing, and prosocial skills

NA

Reinforcement, immediate performance feedback from a mentor, goal setting through missions, modeling, social support, and comparison

No. This game is offered in the home context and can be played by children independently.

No

Adventure, strategy, educational

The player takes the role of a space captain who works for an interplanetary organization in search of rare minerals throughout the universe. The player is assigned various missions and side missions, each with its own adventurous storyline and characters.

During their missions players are confronted with assignments requiring specific skills to solve problems in the areas of time management, planning/organizing, and prosocial functioning.

Players' goal is collecting and recovering rare minerals that lie scattered throughout the universe. During their missions, players have various different tasks they have to complete. There are three minigames with its own specific goals: "Labyrinth," time management; "Explorobot," planning/organizing; and "Space Travel Trainer," prosocial behavior.

Restricted daily play time (45 minutes in main game; 20 minutes in social community)

Complete missions/tasks/levels before moving to the next one

Finishing up assignments within a certain time frame

Communicate in the social community through predefined messages

Exchange earned in-game currency for shop items to upgrade your personal space ship

Achievements, solving puzzles, fetch quests, dialogs with NPCs, rewards, in-game currency, feedback, making short- and long-term appointments with NPCs, strategy use, goal setting, social community, collaborating with NPCs, levels, customizable space ship, collecting items, inventory

This game will enhance skill transference because of the combination of the following elements: theoretical underpinnings, focus on behavior strategies, use of feedback from a mentor, motivation, and reward system.

A futuristic, science fiction game world with multiple planets in different solar systems, each with its own environment

Space captain, male/female, nickname (automatically generated)

Navigate through the game world, talk to NPCs, collect items in the game world, communicate through predefined messages in social community

Computer

NA

20 hours

ADHD, attention deficit/hyperactivity disorder; NA, not applicable; NPC, nonplayable character. 
Table 2. Description of Special Features in the Game

Special feature

Space ship editor

Shops

Inventory

Player profile

\section{Description}

Once the player is able to access his or her space ship, he or she will find the space ship editor. This is an application in the game in which the player is able to customize the ship to his or her liking. Through the game the player will find several items for his or her spaceship, but he or she can also buy items in shops on the different planets.

The player will find several shops on different planets in the game. In those shops he or she is able to purchase and sell items. The player knows which items he or she can afford and will receive descriptions of the items so the player knows more about the item.

In the game the player finds items that can be categorized into minerals, Ico (the robot) upgrade, rocket parts, and items. These items are stored in his or her inventory. The player can use these items in the shops to sell them or in the space ship editor.

The player has a player profile within the game, which includes an avatar picture of the player, together with a list of all the missions the player has completed, and the player's current spaceship is also visible. addition, players learn that it may be helpful to break down an assignment into pieces or to relax before making decisions. The labyrinth game is divided into two different parts. In the first part the player collects minerals in a maze within a limited time frame. In the second part the player estimates the time needed to collect all the minerals. In both parts of the game several strategies can be used to optimize performance, such as (1) the player planning optimal route on a map before entering cave, (2) clicking on the clock to check time, and (3) using the so-called "safe zones." In these zones time pauses so the player can plan his or her next move or just relax. The player has to collect minerals while facing distractions in the maze, thus learning to keep the main goal in mind. For both parts of this minigame there are six different levels, increasing in difficulty. A level is completed when the player collects all the minerals within the restricted time frame or when the player finishes on time (i.e., within his or her estimated time frame).

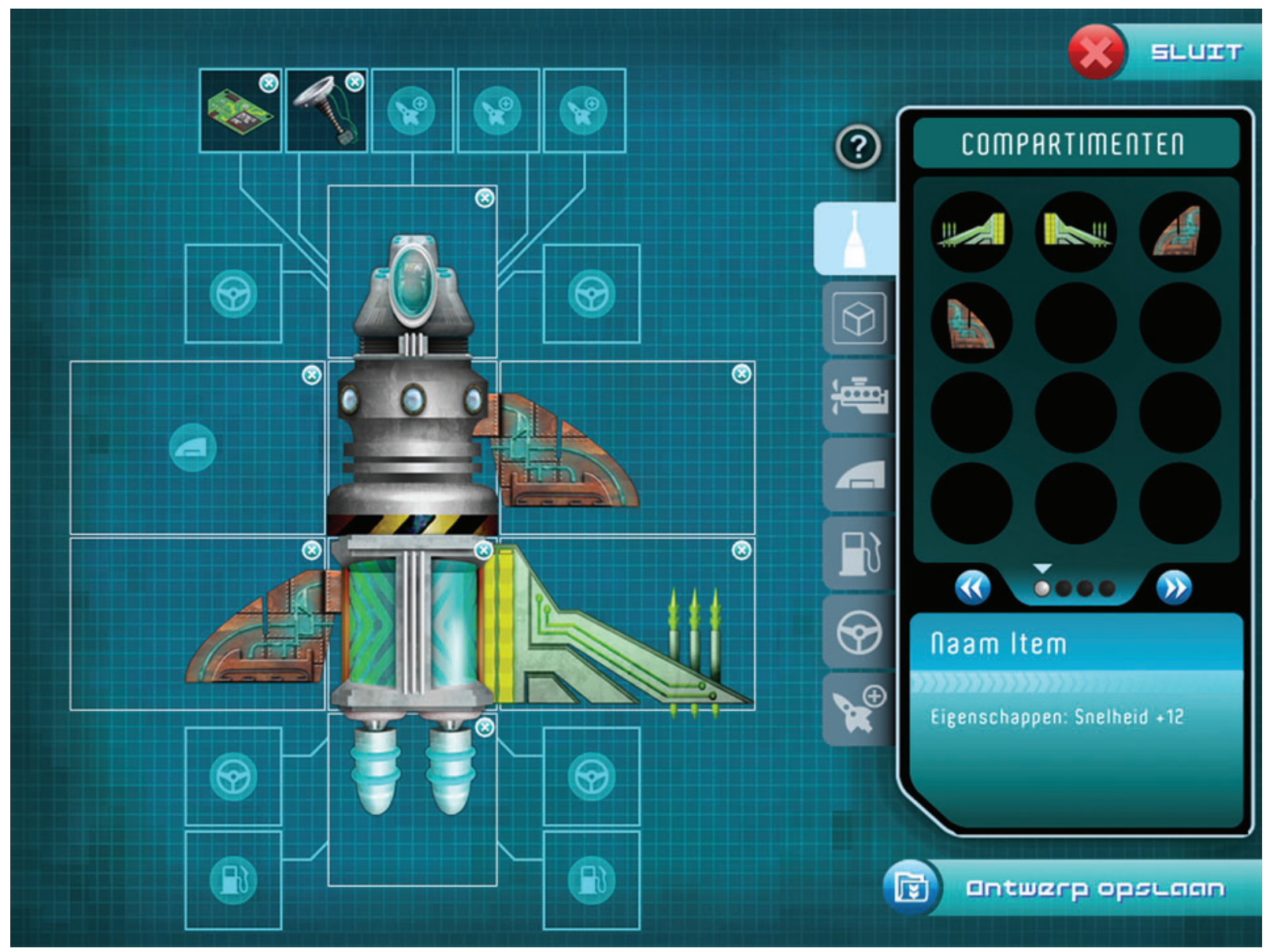

FIG. 2. Space ship editor. (Color graphics available at www.liebertonline.com/g4h) 


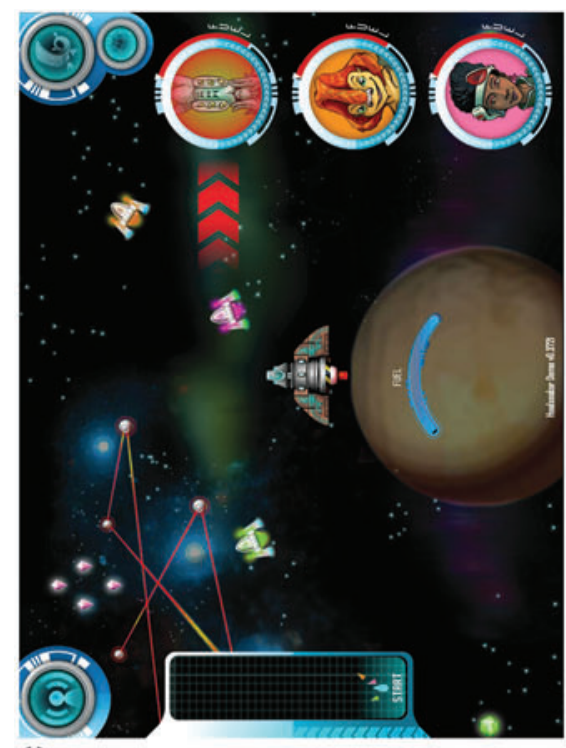

0
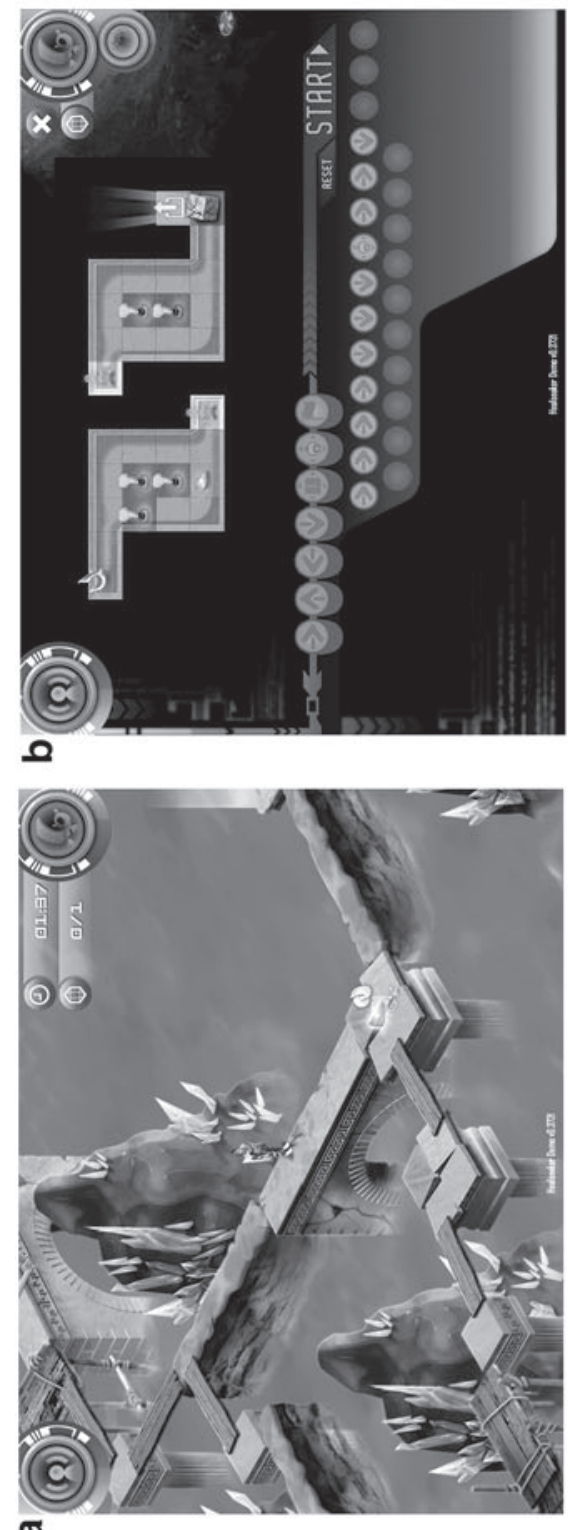

Minigame 2: "Explorobot." Players learn to plan ahead and break down the total assignment into pieces. The player has to collect several minerals that lie scattered in a sewer, using the robot Ico. The player determines the shortest route for Ico and then gives Ico this route description by means of a series of commands. If the player makes a mistake in planning the route, all commands will be deleted, and the player has to plan the route again. As a strategy to optimize performance, players can use checkpoints. If a player makes a mistake after a checkpoint, the robot will jump back to the last checkpoint, and the route can be adjusted from there on. The player can choose to use a limited amount of checkpoints per level. In total, there are 51 levels of difficulty with several tutorial levels. As it may be too hard for some players to find the ideal route, a margin is determined, which is the number of steps needed for the optimal route plus 30 percent (with a maximum of 10 steps). A level is completed when the optimal route (i.e., minimum number of steps) is planned for Ico.

Minigame 3: "Space Travel Trainer." Here players learn to help their team members and to behave in a more prosocial manner toward others. The player flies his or her space ship from planet to planet to reach the target planet with three team members. These team members (named Nika, Vesto, and Kortar) are not real players, but are computer generated, and called nonplayable characters. The team members depend on the player when handling obstacles, such as a star rain, while they follow their predefined route. If the player does not help his or her team members by giving the right commands (e.g., shield, boost, cloak) in time, they inevitably get stuck with low energy levels, which the player has to replenish. Team members ask for help and express their emotions when in dangerous situations. The player can thus use more than one channel to interact with the team members. In total, there are 31 different levels of difficulty. A level is completed when all team members and the player have finished together.

\section{Social community}

To stimulate prosocial behavior, a social community was developed in which players can see each other's profiles and space ships and communicate with each other through predefined messages, for example, with a "thumbs up" or "thank you' button (Fig. 4). In addition, players can see each other's rank and current mission status. This aims to stimulate gameplay and generates some competition between players.

Achievements are related to the learning goals of the intervention and to rewarding players for prosocial behavior within the social community, such as helping other players or giving compliments.

\section{Acceptance and Usability Study}

The initial prototype had three minigames focusing on time management, planning/organizing, and prosocial behavior. The player's mission was to collect as many minerals as possible. The above-mentioned social community, missions, side missions, and special features had not yet been developed. From October 2011 to March 2012 a pilot study was conducted to test the feasibility of conducting a randomized trial on the full game.

As part of the pilot study, participants also filled out questionnaires designed to assess acceptance and usability of 


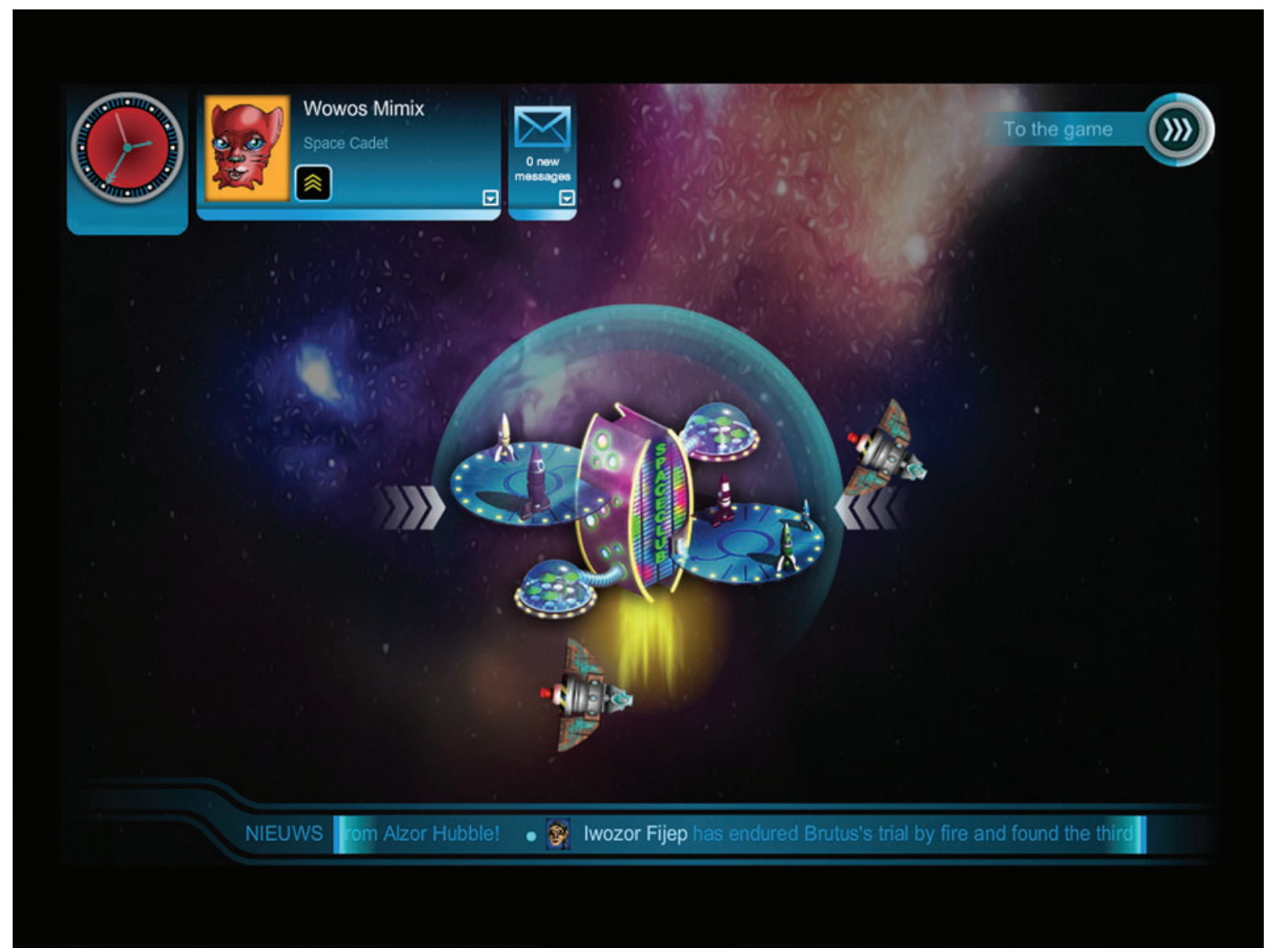

FIG. 4. Social community (called Space Club). (Color graphics available at www.liebertonline.com/g4h)

several game elements. Acceptance and usability were assessed to inform design decisions for further development of the game to a final version to be evaluated in a randomized controlled trial for outcome efficacy.

\section{Participants}

Candidates for the pilot study were identified and informed by their therapist. The therapists were all members of the consortium consisting of ADHD specialized mental healthcare services. Once a potential participant was identified, children and parents received information letters from the researcher, allowing them to make an informed consent about voluntary participation in the pilot study. Inclusion criteria were (a) having a clinical DSM-IV-TR ADHD diagnosis (all subtypes were included) set by a certified healthcare professional, (b) between 8 and 12 years of age, (c) being stable on pharmacological and/or psychological ADHD treatment for at least 2 months prior to baseline assessment (determined by healthcare professionals on the basis of medication data and behavioral observation), and (d) availability of a computer workstation at home with Internet and sound facilities. Children were excluded if they had an estimated total IQ of 70 or lower and had a physical and/or cognitive disability (i.e., deafness, blindness) that would predict great difficulties in playing the serious game and would be problematic for standardized measurements.
In total, 66 children were referred by their therapist and informed about the studies' purposes. The final sample consisted of 42 clinically referred children with a primary diagnosis of ADHD. Children's age ranged from 8 to 11 years with a mean age of 9.4 years. Children participating in the study had average intelligence (mean $=104$; standard deviation $[\mathrm{SD}]=12.3$ ). This was tested with the Wechsler Intelligence Scale for Children III short version. ${ }^{30}$ There was an absence of any neurological, sensory (blindness, deafness), or motor disorder as stated by the clinicians and parents. All children except for two were taking ADHD medication at study entry. Comorbidity of dyslexia was present in four children.

\section{Procedure}

As part of the pilot study we decided to randomize children to one of the two conditions for playing the "Plan-It Commander" prototype. Twenty children were allowed to play the game for a maximum of three times per two weeks. Twentytwo children were asked trying to play the game about eight times per two weeks. However, as there appeared to be no significant differences $(P>0.05)$ among children's and parents' satisfaction scores between the two groups, we decided to present the results for the total group of children. Children played the game at home for 8 weeks, divided into four periods of 2 weeks, with a free choice in playing one of the 
preferred minigames during the last 2 weeks. Every 2 weeks a different minigame was unlocked in predefined order. Children had their own password and ID to log on from their home. Children were asked to play the game for a minimum of 30 minutes and a maximum of 45 minutes each time. Two children were lost to follow-up, and one child dropped out because of acute psychiatric problems.

Ethical approval was obtained from the Committee of Medical Ethics for Mental Health Care in Utrecht, The Netherlands. Written informed consent was obtained from both parents.

Questionnaires were developed especially for this study to assess expectations and satisfaction. Parents filled out questionnaires measuring expectations at baseline (pretest measurement [see Supplementary Data; Supplementary Data are available online at www.liebertonline.com/g4h]) and satisfaction at follow-up (posttest measurement [see Supplementary Data]). Children filled out a questionnaire at follow-up to assess their satisfaction with the prototype “Plan-It Commander" game (see Supplementary Fig. S1).

\section{Pretest parent expectations}

Parents rated their expectations about the game in different domains during pretest measurement (Table 3). Ratings were collected on questionnaires specifically designed for this study (see Supplementary Data). Questionnaires were filled out at the study location on a laptop. Questions included, "How much improvement do you expect with regard to the time management skills of your child?" Parents rated their answers on a 10-point Likert scale in which $1=$ "none" and $10=$ "a lot." Scores from 6 to 10 were combined and interpreted as a positive response. As shown in Table 3, parents had overall high expectations of the game, except where it concerned learning prosocial behavior and reducing ADHD core symptoms. This might be explained by the fact that parents feel prosocial behavior is hard to target in a game. Learning prosocial behavior through a game requires multiplayer options and a different game structure than proposed in this first prototype. ${ }^{31}$ For these reasons, a social community aspect was integrated in the final version of the game. Furthermore, the game was not focused on diminishing ADHD core symptoms but on improving behavioral skills. There-

Table 3. Proportion of Parents Providing Ratings
of Positive Expectations with Regard
to Goals of the Game Expectation $(\mathrm{n}=42)$ positive (percentage)

General learning effect

Improvement of time management Improvement of planning skills Improvement of prosocial skills Improvement of frustration tolerance Reduction of ADHD symptoms

Responses were provided as ratings on a Likert scale ranging from $1=$ "none" to $10=$ "a lot." Participants who provided a rating of 6 or above were categorized as having positive expectations.

$\mathrm{ADHD}$, attention deficit/hyperactivity disorder. fore, lower expectation scores regarding this topic reflect a realistic insight into the capabilities of this game intervention.

\section{Posttest parent satisfaction}

At posttest measurement, parents answered questions on a 10 -point Likert scale $(1=$ "not at all" and $10=$ "totally") concerning parental perceptions about the burden of playing the "Plan-It Commander" game on the child and family. Mean scores ranged from 2.5 to 4.3 , indicating that most parents did not feel offering such game intervention was troubling for the family. Furthermore, results demonstrated that parents were overall positive about the game. Their average overall satisfaction with the game was $6.7(\mathrm{SD}=1.4)$ (on a scale from 1 to 10). In addition, a majority of the parents (88 percent) reported they would recommend the game to other parents. All parents (100 percent) indicated (on a yes/no question) they would like access to the game once further developed. These findings assured us that our current approach was acceptable for parents and helped us in deciding on how and to which degree children should be exposed to the game.

\section{Posttest child satisfaction}

We also asked the children who played the game to rate their game satisfaction in different areas (Table 4). Ratings were collected on a paper-and-pencil questionnaire specifically designed for this study (see Supplementary Fig. S1). Colors and smiley faces were used to highlight the different answer categories on a 5-point Likert scale (from $1=$ "not at all" to $5=$ "very"). Table 4 shows the number (percentage) of children who gave a positive opinion (i.e., a combination of the two highest scores) on the satisfaction questionnaire. Although only 44 percent of the children indicated they were motivated to play the game, 67 percent of the children indicated they had learned from the game, and 77 percent were positive about making the game available for other children with ADHD. A social community, several side missions, and special features were added to the "Plan-It Commander" prototype, making it more attractive and thereby more motivating and challenging for children. This is relevant as

Table 4. Proportion of Children's Ratings of Satisfaction After Playing the Game

\begin{tabular}{lc}
\hline & $\begin{array}{c}\text { Satisfaction } \\
(\mathrm{n}=39) \text { positive } \\
\text { (percentage) }\end{array}$ \\
\hline $\begin{array}{l}\text { Did you like playing the game? } \\
\text { How motivated were you to play } \\
\text { the game? }\end{array}$ & $25(64$ percent) \\
$\begin{array}{l}\text { Would you like to play more often? } \\
\text { How do you feel after finishing the game? }\end{array}$ & 21 (54 percent) \\
$\begin{array}{l}\text { Have you learned anything? } \\
\text { Should other children with ADHD also } \\
\text { be able to play the game? }\end{array}$ & $26(67$ percent) \\
$\begin{array}{l}\text { Would you like to play this game } \\
\text { with friends together? }\end{array}$ & $25(64$ percent) \\
\hline
\end{tabular}

Responses were provided as ratings on a Likert scale ranging from $1=$ "not at all" to $5=$ "very." A combination of the two highest scores were categorized as having a positive opinion.

$\mathrm{ADHD}$, attention deficit/hyperactivity disorder. 
motivation is thought to be an important mediator for changing behavior. 6,31-33

\section{Qualitative user experience}

At posttest measurement, both parents and children answered an open question concerning changes to the game. They provided useful suggestions and recommendations for improvements, such as requests by children for more characters, travel to different planets, and other characters in the game world. Some parents indicated the game could be made more challenging for their child, especially if they already had broad gaming experience. These important responses and feedback were very supportive in finalizing the full game.

\section{Summary and Future Perspectives}

In this article we outlined important aspects of developing a serious game to impact daily life functioning of children with ADHD. We described how developing a serious game is a collaborative project among various experts and users and how that process was carried out in this project. We outlined the theoretical basis for the game as a therapeutic intervention and described how the theory was implemented in various game components. This was followed by a description of the minigames and structural components of the game in which game components were embodied. The information we provided supports the need in the literature on serious games to provide detailed descriptions on the game themselves, theories that guide them, and the components of the game intended to change behaviors that lead to intended positive outcomes. The information provided is also valuable as a description of a method and approach that represents a significant effort to move beyond serious games aimed at improving neurocognitive functioning, but functioning in important domains of daily life for children with ADHD. The description of our development process was supplemented by a presentation of results for parents and child acceptability and usability ratings of a prototype of the game. We discussed the findings in light of their implications for game development.

Overall, the usability findings indicated positive acceptance of this game intervention by children with ADHD and their parents. These preliminary results, based on a prototype version of the final game, directed further development of the game by including several aspects children proposed themselves (e.g., travel to other planets, more characters, special features). Parents' feedback also helped us in making wellinformed decisions about children's play frequency. The advantage of our survey questionnaire approach compared with a more qualitative approach such as a focus group is that the opinions from larger groups of people can be summarized in a standardized way through ratings. A drawback to this approach is that we may have lost the opportunity to gain some important opinions and feedback from participants due to the structured format of the questions and responses. We did, however, also include open-ended questions, which allowed participants to provide their feedback in a less structured approach.

Both parents and children were quite satisfied with the first prototype and indicated they would recommend the game to other parents of children with ADHD. As parents' high expectations might have influenced their ratings, further re- search should try to control for these expectations by including teacher ratings, blinded measures, and more objective measures such as neuropsychological tasks. In the current study, only two children did not use medication as their treatment as usual. It may well be that medication use is a necessary condition for optimal learning from the current intervention. Future research could examine the effects of this game in a nonmedicated sample to further explore the necessity of medication as treatment as usual. With regard to development, it should be considered to extend the game or to create an addon with different learning goals relevant for different age groups. Games could be made more individualized by creating the option to choose learning modules to suit individual developmental trajectories. This project has created a platform from which future goals could be implemented.

Although these first results regarding expectations and satisfaction are promising, a randomized clinical trial is necessary in order to test the effectiveness of this serious game. As serious games become more popular within mental health care, more research is needed on the implementation of such electronic mental health interventions into the primary process of care. This game format presents an alternative to traditional behavioral interventions currently available for children with ADHD that are often presented in school settings by therapists, making them time consuming, costly, and less accessible compared to digital tools. ${ }^{34-36}$

In sum, the description of the approach and process used in developing "Plan-It Commander," along with the usability findings that led back into the development process, provides an example for developing serious games for similar target groups and outcomes. The findings have implications for defining and describing the complex processes of designing and developing serious games that involve collaborations among diverse stakeholder groups that include structured input from target users and family members.

\section{Acknowledgments}

Support for game development and consultancy with regard to the design of the pilot study was provided by Johnson \& Johnson. We thank all the parents and children for their participation in the pilot study. The study was conducted in collaboration with our Partners' Initiative ADHD (PIA) Network partners: Focuz Treatment Centre for Children and Youth in Rotterdam, Maasstad Hospital, and several institutions for child mental health care, namely, GGZ Delfland in Delft, RIAGG in Schiedam and Rotterdam, Kinderpraktijk Zoetermeer in Zoetermeer, and Kinderpraktijk Hillegersberg in Rotterdam.

\section{Author Disclosure Statement}

K.C.M.B. has been paid by Janssen Pharmaceuticals for consultancy and lectures (fees were paid to the institution) but has not been paid by Janssen Pharmaceuticals to perform the pilot study. I.H.A.F., L.J.V., and R.v.d.H. declare no competing financial interests exist. S.V.d.O. has been involved in the development, implementation, and trialing of "Braingame Brian," an executive functioning game training for children with ADHD, and Zelf Plannen (Plan my Life) and Zelf Oplossingen bedenken (Solution focused treatment), two cognitive behavioral planning interventions for adolescents with ADHD. She has no financial interests in 
either of these interventions. She has been a paid consultant for designing a RCT of "Plan-It Commander" (Janssen Pharmaceuticals) and has received speaker's fees from MEDICE and Shire. P.M.K. has been paid by Janssen Pharmaceuticals for consultancy. M.D. has received personal fees from Shire and Neurotech Solutions outside the submitted work. H.J.J.v.O. and A.W. are employees of Janssen Pharmaceuticals. R.v.S. is an employee of RANJ Serious Games. A.M. has been paid by Janssen Pharmaceuticals for consultancy and has been a consultant to, a member of an advisory board of, and/or speaker for Janssen Pharmaceuticals, Eli Lilly, Eurocept, and Neurim Pharmaceuticals in the past 2 years but is not an employee or a stock shareholder of any of these companies.

\section{References}

1. Riper H, Andersson G, Christensen H, et al. Theme issue on e-mental health: A growing field in internet research. J Med Internet Res 2010; 12:e74.

2. Shalini L, Carol EA. E-mental health: A rapid review of the literature. Psychiatr Serv 2014; 65:24-32.

3. Fernandez-Aranda F, Jimenez-Murcia S, Santamaria JJ. Video games as complementary therapy tool in mental disorders: Playmancer, a European multicentre study. J Ment Health 2012; 21:364-374.

4. Parkin A. Computers in clinical practice: Applying experience from child psychiatry. BMJ 2000; 321:615-618.

5. Van der Oord SV, Ponsioen AJ, Geurts HM, et al. Pilot study of the efficacy of a computerized executive functioning remediation training with game elements for children with ADHD in an outpatient setting: Outcome of parent and teacher-rated executive functioning and ADHD behavior. J Atten Disord 2012; 18:699-712.

6. Jaeggi SM, Buschkuehl M, Shah P, Jonides J. The role of individual differences in cognitive training and transfer. Mem Cognit 2014; 42:464-480.

7. Luman M, Oosterlaan J, Sergeant JA. The impact of reinforcement contingencies on AD/HD: A review and theoretical appraisal. Clin Psychol Rev 2005; 25:183-213.

8. Sagvolden T, Aase H, Zeiner P, Berger D. Altered reinforcement mechanisms in attention-deficit/hyperactivity disorder. Behav Brain Res 1988; 94:61-71.

9. Emes C. Is Mr Pac Man eating our children: A review of the effects of videogames on children. Can J Psychiatry 1997; 42:409-414.

10. Tannock R. Television, videogames, and ADHD: Challenging a popular belief. ADHD Rep 1997; 5:3-7.

11. Van der Oord S. Gaming and ADHD: Opportunity or danger? J Pedagogy Child Psychiatry Clin Child Psychol 2012; 37:119-125.

12. Barkley RA. Attention-Deficit Hyperactivity Disorder. A Handbook for Diagnosis and Treatment, 3rd ed. New York; Guilford Press; 2006.

13. Melby-Lervåg $\mathrm{M}$, Hulme $\mathrm{C}$. Is working memory training effective? A meta-analytic review. Dev Psychol 2012; 49: 270-291.

14. Rapport MD, Orban SA, Kofler MJ, Friedman LM. Do programs designed to train working memory, other executive functions, and attention benefit children with ADHD? A meta-analytic review of cognitive, academic and behavioral outcomes. Clin Psychol Rev 2003; 33:1237-1255.

15. Chacko A, Bedard AC, Marks DJ, et al. A randomized clinical trial of Cogmed Working Memory Training in school-age children with ADHD: A replication in a diverse sample using a control condition. J Child Psychol Psychiatry $2014 ; 55: 247-255$.

16. Shipstead Z, Redick TS, Engle RW. Is working memory training effective? Psychol Bull 2012; 138:628-654.

17. Sonuga-Barke EJS, Bitsakou P, Thompson M. Beyond the dual pathway model: Evidence for the dissociation of timing, inhibitory and delay-related impairments in attention-deficit/hyperactivity disorder. J Am Acad Child Adolesc Psychiatry 2010; 49:345-355.

18. Abikoff H, Nisseley-Tsiopinis J, Gallagher R, et al. Effects of MPH-OROS on the organizational, time management and planning behaviours of children with ADHD. J Am Acad Child Adolesc Psychiatry 2009; 48:166-175.

19. Storebø OJ, Skoog M, Damm D, et al. Social skills training for attention deficit hyperactivity disorder (ADHD) in children aged 5 to 18 years. Cochrane Database Syst Rev 2011; (12):CD008223.

20. Conners CK. Core symptoms vs functional outcomes in ADHD: Are they different therapeutic targets? Adv Stud Med 2003; 3:S442-S446.

21. DeSmet A, Van Ryckeghem D, Compernolle S, et al. A meta-analysis of serious digital games for healthy lifestyle promotion. Prev Med 2014; 69:95-107.

22. Baranowksi T, Buday R, Thompson I, Baranowski J. Playing for real. Video games and stories for health-related behavior change. Am J Prev Med 2008; 34:74-82.

23. Kato PM. The role of the researcher in making in serious games for health. In: Arnab S, Dunwell I, Debattista K, eds. Serious Games for Healthcare: Applications and Implications. Hershey, PA: IGI-Global; 2012: 1-40.

24. Wixom BH, Todd PA. A theoretical integration of user satisfaction and technology acceptance. Inf Syst Res 2005; 16:85-102.

25. Markopoulos P, Bekker M. Interaction design and children. Interact Comput 2003; 15:141-149.

26. Cameron LD, Leventhal H. Vulnerability beliefs, symptom experiences, and the processing of health threat information: A self-regulatory perspective. J Appl Soc Psychol 1995; 25:1859-1883.

27. Bandura A. Social Foundations of Thought and Action: A Social Cognitive Theory. Upper Saddle River, NJ: Prentice Hall; 1986.

28. Kato PM, Cole SW, Bradlyn AS, Pollock BH. A video game improves behavioral outcomes in adolescents and young adults with cancer: A randomized trial. Pediatrics 2008; 122:305-317.

29. Beale IL. Video Games for Health. Principles and Strategies for Design and Evaluation. New York: Nova Science Publishers, Inc.; 2011.

30. Wechsler D. Wechsler Intelligence Scale for Children, 3rd ed. San Antonio, TX: Psychological Corporation; 1991.

31. Van Rooij AJ, Jansz J, Schoenmakers TM. What Do We Know About... Effects of Games. A Short Review About Scientific Research to the Effects of Games. Zoetermeer, The Netherlands: Stichting Kennisnet; 2012.

32. Bollar S. Game-Based Learning-Why Does It Work? www .bottomlineperformance.com/gamebasedlearning/ (accessed October 20, 2012).

33. Prins PJM, Dovis S, Ponsioen A, et al. Does computerized working memory training with game elements enhance motivation and training efficacy in children with ADHD? Cyberpsychol Behav Soc Netw 2012; 14:115-122. 
34. Boyer EB, Geurts HM, Prins PJM, Van der Oord S. Two novel CBTs for adolescents with ADHD: The value of planning skills. Eur Child Adolesc Psychiatry 2014 December 31 [Epub ahead of print]. doi: 10.1007/s00787-014-0661-5.

35. Evans SW, Schultz BK, DeMars CE, Davis H. Effectiveness of the challenging horizons after-school program for young adolescents with ADHD. Behav Ther 2011; 42:462-474.

36. Kazdin AE, Blase SL. Rebooting psychotherapy research and practice to reduce the burden of mental illness. Perspect Psychol Sci 2011; 6:21-37.
Address correspondence to: Kim C.M. Bul, MSc Yulius Academy

Yulius Mental Health Care Organization Dennenhout 1

2994 GC Barendrecht, The Netherlands

E-mail: k.bul@yulius.nl 